\title{
Accretion of stellar winds onto Sgr A*
}

\author{
Jorge Cuadra ${ }^{1}$ and Sergei Nayakshin ${ }^{2}$ \\ ${ }^{1}$ Max-Planck-Institut für Astrophysik, D-85741 Garching, Germany \\ ${ }^{2}$ Department of Physics and Astronomy, University of Leicester, LE1 7RH, UK \\ email: jcuadra@mpa-garching.mpg.de, Sergei.Nayakshin@astro.le.ac.uk
}

\begin{abstract}
We report a 3-dimensional numerical study of the accretion of stellar winds onto Sgr $\mathrm{A}^{*}$, the super-massive black hole at the centre of our Galaxy. Compared with previous investigations, we allow the stars to be on realistic orbits, include the recently discovered slow wind sources, and allow for optically thin radiative cooling. We first show the strong influence of the stellar dynamics on the accretion onto the central black hole. We then present more realistic simulations of Sgr A* accretion and find that the slow winds shock and rapidly cool, forming cold gas clumps and filaments that coexist with the hot X-ray emitting gas. The accretion rate in this case is highly variable on time-scales of tens to hundreds of years. Such variability can in principle lead to a strongly non-linear response through accretion flow physics not resolved here, making Sgr A* an important energy source for the Galactic centre.
\end{abstract}

Keywords. Galaxy: center - black hole physics

\section{Introduction}

Sgr A* is identified with the $M_{\mathrm{BH}} \sim 3.5 \times 10^{6} M_{\odot}$ super-massive black hole (SMBH) at the centre of our Galaxy (Schödel et al. 2002). By virtue of its proximity, Sgr A* plays a key role in the understanding of Active Galactic Nuclei (AGN). Indeed, this is the only $\mathrm{SMBH}$ where observations detail the origin of the gas in its vicinity. This information is absolutely necessary for the accretion problem to be modelled self-consistently.

One of Sgr A* puzzles is its low luminosity with respect to estimates of the accretion rate. Young massive stars in the inner parsec of the Galaxy fill this region with hot gas. From Chandra observations, one can measure the gas properties around the inner arcsecond (one arcsecond, $1^{\prime \prime}$, corresponds to $\sim 0.04 \mathrm{pc}, \sim 10^{17} \mathrm{~cm}$, or $\sim 10^{5} R_{\mathrm{S}}$ for Sgr $A^{*}$ ) and then estimate the Bondi accretion rate (Baganoff et al. 2003). The expected luminosity is orders of magnitude higher than the measured $\sim 10^{36} \mathrm{erg} \mathrm{s}^{-1}$.

The hot gas, however, is continuously created in shocked winds expelled by the stars near Sgr $A^{*}$, and the stars themselves are distributed in discs (Paumard et al. 2006). The situation then is far more complex than in the idealised Bondi model. An alternative approach is to model the gas dynamics of stellar winds, assuming that the properties of the wind sources are known (Coker \& Melia). Here we present our numerical modelling of wind accretion onto Sgr $\mathrm{A}^{*}$, the first to allow the wind-producing stars to be on Keplerian orbits.

\section{Numerical approach and first results}

We use the smooth particle hydrodynamics (SPH) code GADGET-2 (Springel 2005) to simulate the dynamics of stars and gas in the gravitational field of the SMBH. To model the stellar winds, new gas particles are continously created around the stars. The SMBH is modelled as a 'sink' particle (Springel et al. 2005), with all the gas passing within $0.05^{\prime \prime}$ from it disappearing from the computational domain. In addition, we eliminate all the 
gas that goes beyond a $20^{\prime \prime}$ outer boundary. More details of the numerical method along with validation tests have been presented elsewhere (Cuadra et al. 2006).

We first performed several simulations with different configurations for the stellar orbits. We found that the angular momentum of the gas has a strong dependence on the orbital motions. Moreover, only the fraction of the gas with the 'right' low angular momentum goes to the inner region and can be accreted. Therefore, when the stars are rotating in a disc, the gas has more angular momentum and the accretion rate is lower than in the case where stars orbit the central black hole isotropically (Cuadra et al. 2006). This result highlights the necessity of using realistic orbits to model the accretion onto Sgr $A^{*}$.

\section{Set-up of the new simulations}

In our previous calculations (Cuadra et al. 2006), we used an ensemble of stars in circular orbits with roughly the same properties as the observed population. For the new simulations presented here, we use the latest available data for individual stars, going a step forward on the creation of more realistic models.

We include in our calculations the 30 stars identified as WRs or LBVs (Paumard et al. 2006). For the initial conditions of the stellar winds, we use the wind velocities and mass loss rates calculated by Martins et al. (in preparation). For the rest of the stars we use the wind velocities derived previously (Paumard et al. 2001) when available, otherwise we set $v_{\mathrm{w}}=1000 \mathrm{~km} \mathrm{~s}^{-1}$. The mass loss rate for the stars without data is set to a common value such that the total mass loss rate from all the stars is $10^{-3} M_{\odot} \mathrm{yr}^{-1}$. More tests changing the mass loss rate of the stars are discussed by Cuadra et al. (in prep.).

We use the published positions and velocities for the mass-losing stars (Paumard et al. 2006). For the stars without a $z$ coordinate, this is set assuming that the star is in the corresponding disc. With the current $3 \mathrm{~d}$ positions and velocities for the stars, we integrate the orbits back in time for $\sim 1200 \mathrm{yr}$. The positions and velocities at this time were used as initial conditions for the SPH simulations.

\section{Two-phase gas}

Figure 1 shows the resulting morphology of the gas at the end of one simulation. Cool dense regions in the gas distribution are mainly produced by winds from LBVs. When shocked, these slow winds attain a temperature of only around $10^{6} \mathrm{~K}$, and, given the high pressure environment of the inner parsec of the GC, quickly cool radiatively (Cuadra et al. 2005). LBV winds form bound clouds of gas, often flattened into filaments due to the SMBH potential. On the other hand, the WRs by themselves do not produce much structure. The fast winds they emit have temperatures $>10^{7} \mathrm{~K}$ after shocking, and do not cool fast enough to form clumps. This temperature is comparable to that producing X-ray emission detected by Chandra. Gas cooler than that would be invisible in X-rays due to the finite energy window of Chandra and the huge obscuration in the Galactic plane.

We find that it is harder to form a disc-like structure like the one found in our previous studies (Cuadra et al. 2006). The main reason for this is that in the present simulations there is not as much cold gas. There are less LBVs - from whose winds the cold clumps are mostly formed - and they have smaller mass loss rates than previously assumed. Moreover, while before we put most slow wind stars in the same plane, now we put only two out of the three innermost stars in one plane, so in this case the preference for one plane is lower. 

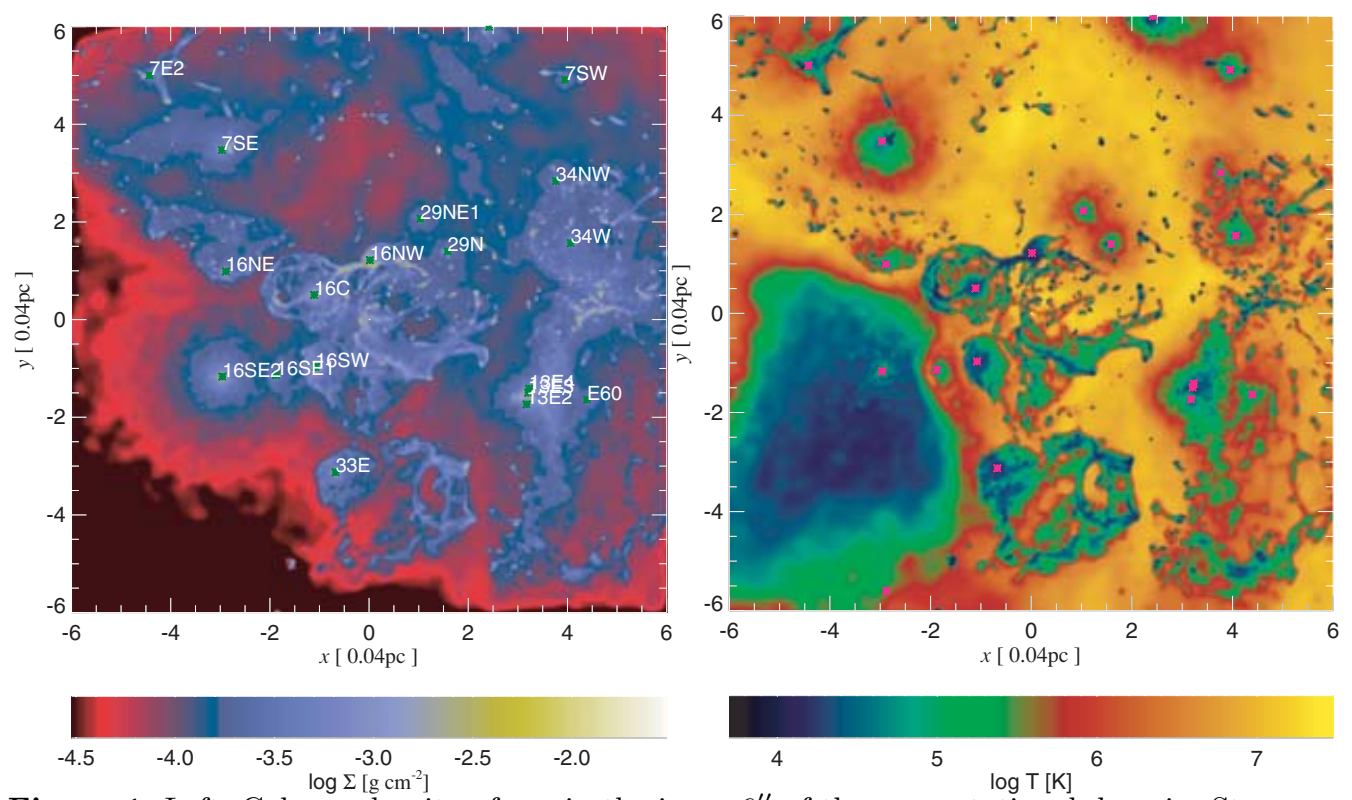

Figure 1. Left: Column density of gas in the inner $6^{\prime \prime}$ of the computational domain. Stars are shown as green symbols with labels. Right: Averaged temperature of the same region. Dense cold clumps form around the IRS 16 and 13 groups, where slow winds from different stars collide. Winds from the WR stars also remain cold but diffuse before interacting with other winds.

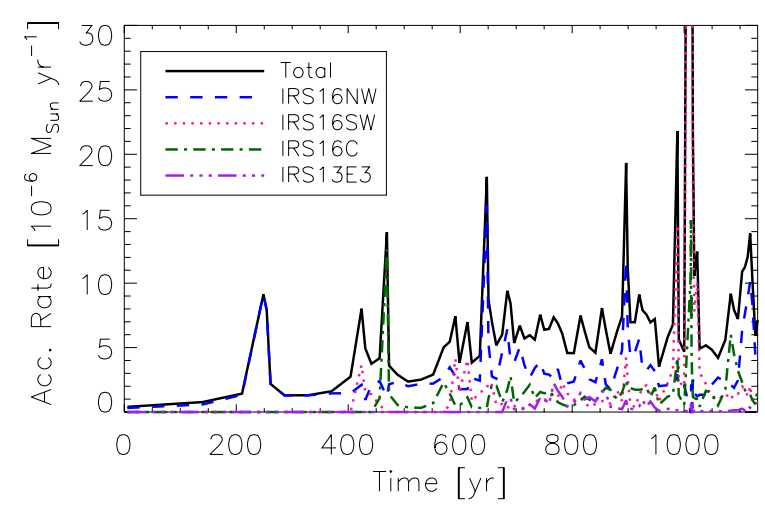

Figure 2. Accretion history from one of our simulations. Different lines show the contribution of material coming from a few selected stars. Notice that most of the accreted matter comes from the stars in the IRS 16 group.

\section{Accretion onto Sgr A*}

From any of our simulations, we can extract the accretion history onto Sgr A* Accretion is here defined by the quantity of gas entering the inner boundary $\left(0.05^{\prime \prime}\right)$ of our computational domain.

In all the simulations the accretion rate is quite variable, changing by factors $\sim 10$ on timescales as short as the chosen resolution of $10 \mathrm{yr}$. The variability is mainly caused by the accretion of clumps originating from material produced by the few innermost slow wind stars in the IRS 16 group (see Fig. 5). But even if the mass loss rates from these stars are reduced by more than a factor 10, a large degree of variability remains (see Cuadra et al., in prep.).

In our previous work, we had found that the accretion onto the SMBH could be separated into a quasi-steady hot component and a variable cold one coming from the episodic 
accretion of clumps. In these new calculations, we find instead that most of the accreted material is actually hot $\left(T>10^{7} \mathrm{~K}\right)$, regardless of which star it is coming from. This is most likely the effect of the eccentric orbits - they increase the total velocity of the wind, making it acquire a large kinetic energy which is later thermalized.

While we cannot resolve the inner accretion flow to predict the actual accretion rate onto Sgr A*, we estimate that variability by a factor of a few should still reach the black hole. In the extremely sub-Eddington regime of Sgr $\mathrm{A}^{*}$, a small change in the accretion rate produces a non-linear response on the luminosity (Yuan et al. 2002). The results from our simulations, the observational evidence for higher luminosity in the recent past (Revnivtsev et al. 2004), and the idea of star formation in an AGN-like accretion disc a few million yr ago (Nayakshin \& Cuadra 2005), all suggest that on long time-scales Sgr $A^{*}$ is an important energy source for the inner Galaxy.

\section{Conclusions}

The gas at $r \sim 1^{\prime \prime}$ distances from Sgr A* has a two-phase structure, with cold filaments immersed into hot X-ray emitting gas. Both the fast and the slow phase of the winds contribute to the accretion flow onto Sgr $\mathrm{A}^{*}$. The accretion rates we obtain are of the order of a few times $10^{-6} \mathrm{M}_{\odot}$ year ${ }^{-1}$, consistent with the Chandra estimates, although very variable on time-scales as short as tens of years. This implies that the current very low luminosity state of Sgr A* may be the result of a relatively unusual quiescent state.

\section{Acknowledgements}

JC thanks the IAU and the ASCR for their financial support to attend the meeting.

\section{References}

Baganoff, F. K. et al. 2003, ApJ, 591, 891

Coker, R. F. \& Melia, F. 1997, ApJL, 488, L149

Cuadra, J., Nayakshin, S., Springel, V. \& Di Matteo, T. 2005, MNRAS, 360, L55

Cuadra, J., Nayakshin, S., Springel, V. \& di Matteo, T. 2006, MNRAS, 366, 358

Nayakshin, S. \& Cuadra, J. 2005, A\&A, 437, 437

Paumard, T., Maillard, J. P., Morris, M. \& Rigaut, F. 2001, å, 366, 466

Paumard, T. et al. 2006, ApJ, 643, 1011

Revnivtsev, M. G. et al. 2004, A\&A, 425, L49

Schödel, R. et al. 2002, Nature, 419, 694

Springel, V. 2005, MNRAS, 364, 1105

Springel, V., Di Matteo, T. \& Hernquist, L. 2005, MNRAS, 361, 776

Yuan, F., Markoff, S. \& Falcke, H. 2002, A\&A, 383, 854

THIERry Courvoisier: It seems very striking that there is no substantial net angular momentum. How does matter present in the center gets rid of angular momentum?

Jorge CuAdra: I guess you refer to the process that led the material that formed the stars to the inner parsec. This is an important issue but I do not think the answer to your question is known. It is possible that the gas originated in a collision between molecular clouds with opposite angular momentum for instance. 